\title{
AN OPERATOR EQUATION AND RELATIVISTIC ALTERATIONS IN THE TIME FOR RADIOACTIVE DECAY
}

\author{
ROBERT A. HERRMANN
}

Mathematics Department United States Naval Academy 572 Holloway Rd.

Annapolis, MD 21402-5002 USA

(Received September 29, 1994)

\begin{abstract}
In this paper, using concepts from the nonstandard physical world, the linear effect line element is derived. Previously, this line element was employed to obtain, with the exception of radioactive decay, all of the experimentally verified special theory relativistic alterations in physical measures. This line element is now used to derive, by means of separation of variables, an expression that predicts the same increase in the decay time for radioactive material as that predicted by the Einstein time dilation assumption. This indicates that such an increase in lifetime can be attributed to an interaction of the radioactive material with a nonstandard electromagnetic field rather than to a basic time dilation.
\end{abstract}

KEY WORDS AND PHRASES. Special relativity, separation of variables, radioactive decay, time dilation, nonstandard analysis, nonstandard electromagnetic field.

1992 AMS SUBJECT CLASSIFICATION CODES. 83A05, 03H10.

\section{INTRODUCTION.}

In [7], a specific operator equation related to a partial differential equation, the Schwarzschild and linear effect line elements, and the concept of separation of variables are used to derive various relativistic alteration expressions. As discussed in [6], the measurable aspects of the special theory of relativity need not be related to the so-called general time dilation or length contraction concepts. Indeed, under the assumption that there exists a nonstandard substratum - a nonstandard electromagnetic field (NSEM field) - it is conjectured that all of the special theory predicted alterations in measured quantities can be obtained by means of a electromagnetic propagation theory. The quantities would be measured by infinitesimal light-clocks and, in order to avoid the model theoretical error of generalization, no other instrumentation would be allowed.

In [7], with the exception of relativistic alterations in the lifetime of radioactive material, all of the major special theory relativistic alterations in measured quantities are obtained from the linear effect line element without the introduction of Einstein universal time dilation or length contraction. What is derived first is a statement relating infinitesimal time measurements, where these measurements are interpreted as changes in the number of counts or "ticks" that 
occur within a measuring infinitesimal light-clock. The use of infinitesimal light-clocks and only infinitesimal light-clocks to obtain the experimentally verified alterations indirectly implies that such alternations may be caused by an electromagnetic interaction with the substratum (emis) rather than an Einstein time dilation.

The main purpose of this paper is to present a derivation of the linear effect line element using substratum theory and to use the methods in [7] to predict the experimentally verified [1] relativistic alteration in radioactive decay time.

\section{THE LINEAR EFFECT LINE ELEMENT.}

In what follows, we are considering a standard set-theoretic superstructure $\mathcal{M}=\langle\mathbb{R}, \in,=\rangle$ with ground set the real numbers $\mathbb{R}$ and this structure is embedded into a nonstandard elementary extension ${ }^{*} \mathcal{M}=\left\langle{ }^{*} \mathbb{R}, \in, \Rightarrow\right\rangle$ that is an enlargement [9]. In [5], the chronotopic interval (i.e. proper time interval) is derived and yields the following interpretation. An infinitesimal light-clock is in linear and uniform motion, and $L$ is an infinitesimal number representing twice the length of the arm. The infinitesimal light-clock has "ticked" $\Pi_{m} \in \mathbb{N}_{\infty}$ times, where $\mathbb{N}_{\infty}$ is the set of Robinson infinite natural numbers. The apparent natural world linear distance traversed by the electromagnetic radiation in its to-and-fro motion within the moving infinitesimal light-clock is st $\left(L \Pi_{m}\right)$.

At a spacetime point within the substratum there are four infinitesimal light-clocks that are considered as stationary. These four infinitesimal light-clocks are used as models for the Einstein measurement [8] of the relative velocity of the moving light-clock. Three of these "clocks" are corresponded to a Cartesian coordinate system used to model the Einstein length measurements and the fourth infinitesimal light-clock is used to measure the Einstein time. The measurements taken by the stationary infinitesimal light-clocks are the "ticks" that have occurred within these light-clocks relative to the "ticks" that have occurred in the moving infinitesimal light-clock. Let $\omega \in \mathbb{N}_{\infty}$. Then as proved in $[4$, p. 108] for each $r \in \mathbb{R}$, there exists an $x \in\{m / \omega \mid$ ( $m \in$ $\left.\left.{ }^{*} \mathrm{Z}\right) \wedge\left(|m|<\omega^{2}\right)\right\}$ such that $x \approx r$. As discussed in [6] and due to this result, the use of infinitesimal light-clocks allows for time and length measurements to be considered as varying over intervals of real numbers. This might be expressed by saying that they can take on a "continuum" of values. Thus standard analysis can be applied.

Let the 4-tuple $\left(x^{s}, y^{s}, z^{s}, t^{s}\right)$ be the standard part of the changes in the four stationary infinitesimal light-clock measurements used to calculate the Einstein relative velocity over the "tick" period $\Pi_{m}$. This means that as far as the moving infinitesimal light-clock is concerned the number of "ticks" between the emission, from a substratum stationary source located at a point that is not necessarily on the line of motion, of a relative velocity measuring light pulse and its being receive back by the source is $\Pi_{m}$. The expression derived in [5] is

$$
\left(\mathrm{st}\left(L \Pi_{m}\right)\right)^{2}=\left(t^{s}\right)^{2} c^{2}-\left(\left(x^{s}\right)^{2}+\left(y^{s}\right)^{2}+\left(z^{s}\right)^{2}\right),
$$

where $c$ is the to-and-fro measurement of the velocity of electromagnetic radiation.

There exist hypernatural numbers in $\mathbb{N}_{\infty}$ such that $L \Pi_{m}$ and the $x^{s}, y^{s}, z^{s}, t^{s}$, prior to extracting the standard part, are all nonzero infinitesimal numbers. This allows for the infinitesimalizing of expression (2.1) and yields

$$
(d S)^{2}=\left(d t^{s}\right)^{2} c^{2}-\left(\left(d x^{s}\right)^{2}+\left(d y^{s}\right)^{2}+\left(d z^{s}\right)^{2}\right) .
$$


Also, due to the freedom allowed for interpretation of these infinitesimal quantities when they are used to measure an effect over a standard natural world interval [2, p. 175], it can be assumed that $(d S)^{2}=\left(L \Pi_{m}\right)^{2}$. Expression (2.2) is that which is used when (2.1) is extended so as to include nonuniform substratum behavior. We often write $\left(d r^{s}\right)^{2}=\left(d x^{s}\right)^{2}+\left(d y^{s}\right)^{2}+\left(d z^{s}\right)^{2}$.

Expression (2.2) relates behavior of infinitesimal light-clock counts but does not indicate that there might be a cause for such behavior. Distinct from the classical approach to the special theory, a cause can be postulated relative to the behavior of a NSEM field. In what follows, the timing infinitesimal light-clocks are used as an analogue model to investigate how oscillatory NSEM field behavior is altered by an approximately static and a nonreversible process denoted by $P$. The process $P$ is isotropic and related to motion viewed from points in Euclidean space. Suppose that the counts of these measuring infinitesimal light-clocks are affected by this $P$-process. We seek a relationship $\phi\left(d x^{m}, d y^{m}, d z^{m}, d t^{m}\right)$ or $\Phi\left(d r^{m}, d t^{m}\right)$ between these altered count changes as they would appear on the measuring infinitesimal light-clocks so that $L \Pi_{m}=\phi\left(d x^{m}, d y^{m}, d z^{m}, d t^{m}\right)$ or $L \Pi_{m}=\Phi\left(d r^{m}, d t^{m}\right)$. Assume that an electromagnetic pulse is emitted from a source that exhibits a scalar absolute substratum or relative velocity $v$ over an infinitesimal light-clock measured time interval $d t^{\boldsymbol{t}}$, where the subscripts or superscripts $s$ indicate no effect produced by $P$ and a subscript or superscript $m$ indicates that there is an effect produced by $P$.

Following [6], let $v$ and $c$ behave within a monadic neighborhood as if they are constant with respect to $P$. Within an NSEM field monadic neighborhood, the scalar Galilean (ballistic) distance traversed by the pulse is

$$
(v+c) d t^{s}=v d t^{s}+c d t^{s}=d r^{s}+d T^{s}, d r^{s}=v d t^{s}, d T^{s}=c d t^{s} .
$$

Recall that the monadic ballistic behavior of the pulse and the nonballistic behavior derived in [6] for the natural world indicate the dual nature of electromagnetic radiation. Suppose that, due to $P$ interacting with the substratum, a smooth microeffect [3] - a kind of damping of the light-clock counts - alters (2.3). This alteration is characterized by the presence of an $\alpha$ in equation (A): $d r^{m}=d r^{s}+\alpha d T^{s}$. Let (B): $d T^{s}=\beta d r^{m}+d T^{m}, d T^{m}=c d t^{m}$ be a corresponding reciprocal expression, where $\alpha, \beta$ are to be determined. Since the effects are to be observed in the natural world, $\alpha, \beta$ have standard values. In order to incorporate the nonreversible nature of the $P$ effects into (2.3), the infinitesimal expression $d r^{s}$ is not reversible in the sense that once it is used for a $P$ effect the substitution back to the form $v d t^{s}$ is not allowed. This is not the case when infinitesimals are considered in a derivative form that has a real standard part. Of course, all measures, except for $v$, are considered as Einstein measures as modeled by infinitesimal light-clock counts.

From (A), (B), we have (C): $d r^{s}=(1-\alpha \beta) d r^{m}-\alpha d T^{m}$. Substituting into (2.2) yields

$$
\begin{gathered}
d S^{2}=\left(1-\alpha^{2}\right)\left(d T^{m}\right)^{2}+2\left(\alpha+\beta\left(1-\alpha^{2}\right)\right) d r^{m} d T^{m}+ \\
\left(\beta^{2}-(1-\alpha \beta)^{2}\right)\left(d r^{m}\right)^{2}
\end{gathered}
$$

The simplest real world aspect of time interval measurement that assumes that timing counts can be added or subtracted is transferred to a monadic neighborhood and requires $d T^{m}$ to take on positive or negative infinitesimal values. Assuming the effects of $P$, as incorporated in $d S^{2}$, 
are independent of whether such a defined infinitesimal light-clock count predicts future behavior or is a consequence of past behavior, then $d S^{2}$ should not alter its value when $d T^{m}$ is positive or negative. This implies that $2\left(\alpha+\beta\left(1-\alpha^{2}\right)\right)=0$. Since we assume that $\alpha$ is a special type of damping of the light-clock counts, for simplicity of calculation, let $\alpha=-\sqrt{1-\eta}$. Hence, $\beta=\sqrt{1-\eta} / \eta$. Substituting into (B) and $(\mathrm{C})$ yields

$$
\begin{aligned}
& d r^{s}=\frac{1}{\eta} d r^{m}+\sqrt{1-\eta} d T^{m} \\
& d T^{s}=\frac{\sqrt{1-\eta}}{\eta} d r^{m}+d T^{m} .
\end{aligned}
$$

Combining both equations in (2.5) produces

$$
\frac{d r^{s}}{d T^{s}}=\frac{\frac{1}{\eta} \frac{d r^{m}}{d T^{m}}+\sqrt{1-\eta}}{\frac{\sqrt{1-\eta}}{\eta} \frac{d r^{m}}{d T^{m}}+1}
$$

To incorporate the requirement that $P$ is static with respect to motion within a monadic neighborhood, we assume that at some spacetime point, including a possible point at infinity, that ' an initial condition is that $d r^{m} / d T^{m}=0$. Using $d r^{s} / d T^{s}=v / c,(2.6)$ yields that $v / c=\sqrt{1-\eta}$ or $\eta=1-v^{2} / c^{2}=\lambda \neq 0$. We note that using $\alpha=\sqrt{1-\eta}$ yields the contradiction $v / c<0$ for the case that $0<v<c$. By substituting $\eta$ into (2.5) and then (2.5) into (2.2) (see (2.4)), we have, where $d T^{m}=c d t^{m}$, the linear effect line element

$$
d S^{2}=\lambda\left(c d t^{m}\right)^{2}-(1 / \lambda)\left(d r^{m}\right)^{2}
$$

This linear effect line element yields a special theory line element if $v=v_{E}$ (the Einstein measure of the relative velocity) and correlates special theory effects to a describable effect via a nonreversible $P$-process. The right hand side of (2.7) represents the necessary alteration in the light-clocks of the measuring infinitesimal light-clocks that are now affected by the $P$-process. The left hand side, $d S^{2}$, is invariant not in the sense of Riemannian geometry but in the sense that it is equal to $\left(L \Pi_{m}\right)^{2}$, where $\Pi_{m}$ is the change in the infinitesimal light-clock counts for the one infinitesimal light-clock being affected by the $P$-process. Since the line element is a relation between infinitesimal quantities, the expression "linear effect" is not intended to imply that the (standard) path of motion of the analogue infinitesimal light-clock is necessarily linear.

Following the usual practice for decay purposes, it is assumed that the atomic structure is momentary at rest when decay occurs. This is modeled by letting $d r^{s}=0$ in (2.2) and $d r^{m}=0$ in (2.7). Hence we have that $\gamma d t^{m}=d t^{s}$, where $\gamma=\sqrt{\lambda} \neq 0$. This does not indicate a change in the concept of time. As implied in [6] and shown in [5], $d t^{m}=u \Pi_{m}, d t^{s}=u \Pi_{s}$. The NSPworld time unit $u$ is not altered but the infinite count number $\Pi_{s}$ has been altered by the factor $1 / \gamma$. The alteration in the infinite count number can be conceived of as being produced by a change in the (linear) length of the nonlinear (osculatory) NSP-world path of motion of the light pulse in the infinitesimal light-clock, where the natural world path of motion appears to be linear. The osculations are damped yielding a shorter path in the NSP-world and, hence, a larger count number $\Pi_{m}$. Using this analogue approach, if the verified alterations in decay time can be predicted, then this would indicate that decay itself is an (emis) effect. Further, the 
result $\gamma d t^{m}=d t^{s}$ is independent of the path of motion of the analogue infinitesimal light-clock. [Much of the procedure outlined in this section can be used to obtain the Schwarzschild and other well-known line elements.]

\section{THE DERIVATION OF THE DECAY PREDICTION.}

The method of separation of variables as used in [7] is the consistent underlying procedure used to predict the verified alteration in decay time. Unfortunately, in [7] a confusing typographical error occurs in expressions (3.2) and (3.3). The symbols $h\left(r^{*}, t^{*}\right)$ should be replaced with $h\left(r^{0}\right)$ and $h\left(r^{m}, t^{m}\right)$ should be replaced with $h\left(r^{m}\right)$. More importantly, the actual decay differential equation used to predict decay time contains a logical difficulty.

Let $N\left(t^{*}\right)$ denote the number of radioactive entities at the light-clock count time $t^{s}$ and $\tau_{s}$ be the (mean) lifetime assumed to be a universal constant. This means that $\tau_{s}$ is not dependent upon Einstein (infinitesimal light-clock) measures of spacetime coordinates at an instant of substratum spacetime. [Note that Einstein uniform relative velocity is a universal constant.] The basic equation is $(-\tau) d N / d t=N$. Technically, this expression can only have meaning if it is assumed that $N$ is a continuous function defined on some time interval. The definition of $N$ indicates that $N \geq 0$, is integer valued and is decreasing. This difficulty can be corrected by using Theorem 4.1 in [3]. Let $[a, b], a>0$, be an appropriate time interval. There exists a ${ }^{*}$-differentiable $\mathcal{N}$ defined on ${ }^{*}[a, b]$ such that $\operatorname{st}(\mathcal{N})=N$ is the number of radioactive entities that exist during an appropriate portion of $[a, b]$ and the finite range of $\mathcal{N}={ }^{*}[g, h]$, where $g$ is the (maximum) (standard) number of radioactive entities when $t^{s}=a$ and $h$ is the (standard) number of radioactive entities remaining when $t^{s}=b$. In the following derivation, the function $\mathcal{N}$ is employed although the solution can be restricted to the less than satisfactory function $N$ by $\operatorname{simply}$ replacing $\mathcal{N}$ with $N$ and removing the ${ }^{*}$ and the st notation.

The operator expression $D(T)=k(\partial T / \partial t)$ as it appears in [7] is extended to an internal and partially differentiable function $T$, where $k$ is a universal constant. This yields

$$
\left({ }^{*} D\right)(T)={ }^{*} k^{*}(\partial / \partial t)(T) .
$$

Define internal and partially differentiable $T(r, t)=\left(0 \cdot r^{2}+1\right) \mathcal{N}(t)$, where $r^{2}=x^{2}+y^{2}+z^{2}$, and let ${ }^{*} D$ be the extended identity map ${ }^{*} I$. Clearly, by *-transfer, ${ }^{*} I$ is separating and the identity on the temporal function. Also, with respect to the measuring instruments, $T\left(r^{s}, t^{s}\right)=\left(0 \cdot\left(r^{*}\right)^{2}+\right.$ 1) $\mathcal{N}\left(t^{s}\right)=\mathcal{N}\left(t^{s}\right)$ and $T\left(r^{m}, t^{m}\right)=\overline{\mathcal{N}}\left(t^{m}\right)$. We show that $T$ is a ${ }^{*}$-universal function. The function $\mathcal{N}$ has the property that for an appropriate $t^{s} \in[a, b], \operatorname{st}\left(\mathcal{N}\left(t^{s}\right)\right)$ is the number of radioactive physical entities present. This number of physical entities also occurs at a substratum time $t$. The number is assumed not to change simply because it is now being counted at the corresponding infinitesimal light-clock time $t^{m} \in[c, d]$. Hence the value $\operatorname{st}\left(\mathcal{N}\left(t^{s}\right)\right)=\operatorname{st}\left(\overline{\mathcal{N}}\left(t^{m}\right)\right)$. From the definition [3] of such functions as $\mathcal{N}$, this implies that $\mathcal{N}\left(t^{s}\right)=\overline{\mathcal{N}}\left(t^{m}\right)$, where $t^{s} \in{ }^{*}[a, b]$ and corresponding $t^{m} \in{ }^{*}[c, d]$. As done previously [7], coordinate transformations are not considered only the language of "measurement" is being used.

Applying (3.1) to $T$, the ${ }^{*}$-transfer of the decay rate equation and the chain rule, one obtains that

$$
\begin{gathered}
\mathcal{N}\left(t^{s}\right)=\left(-{ }^{*} \tau_{s}\right){ }^{*}\left(d / d t^{s}\right) \mathcal{N}\left(t^{s}\right)=\left(-{ }^{*} \tau_{s}\right){ }^{*}\left(d / d t^{m}\right) \overline{\mathcal{N}}\left(t^{m}\right){ }^{*}\left(d / d t^{s}\right)\left(t^{m}\right)= \\
\left(-{ }^{*} \tau_{s} /{ }^{*} \gamma\right)^{*}\left(d / d t^{m}\right) \overline{\mathcal{N}}\left(t^{m}\right) .
\end{gathered}
$$


With respect to $m$,

$$
\overline{\mathcal{N}}\left(t^{m}\right)=\left(-{ }^{*} \tau_{m}\right)^{*}\left(d / d t^{m}\right) \overline{\mathcal{N}}\left(t^{m}\right) .
$$

Using the finite $(\not \approx 0)$ character of the hyperreal numbers being considered, then the standard part of (3.2) and (3.3) yields that $\operatorname{st}\left({ }^{*} \tau_{m}\right)=\tau_{m}=\operatorname{st}\left({ }^{*} \tau_{s} /{ }^{*} \gamma\right)=\tau_{s} / \gamma$. This is the exact expression that appears in [1], where the $\gamma$ that appears in [1] is the reciprocal of the $\gamma$ used in the above derivation. The $\tau_{s}$ can always be taken as the time of decay as measured at rest in the laboratory since the relative velocity of the radioactive entities is determined by experimental equipment that is at rest in the laboratory. Finally, although it may be necessary when arguing for the acceptance of a particluar operator equation as a physical model that $k$ is considered to be either an absolute or universal constant, in this derivation and those that appear in [7] this restriction is unnecessary. Clearly, if such a constancy is not assumed, then the results would be more difficult to interpret.

\section{REFERENCES}

1. BAILEY, J. and et al. Measurements of relativistic time dilatation for positive and negative muons in a circular orbit, Nature, 268(July 1977), 301-305.

2. HERRMANN, R.A. Supernear functions, Math. Japonica, 30(1985), 125-129.

3. HERRMANN, R.A. Fractals and ultrasmooth microeffects, J. Math. Phys., 30(April 1989), 805-808.

4. HERRMANN, R.A. Ultralogics and More, IMP, P. O. Box 3268, Annapolis, MD 21403-0268, 1993.

5. HERRMANN, R.A. Constructing Logically Consistent Special and General Theories of Relativity, Math. Dept., U.S. Naval Academy, Annapolis, MD, 1993.

6. HERRMANN, R.A. Special relativity and a nonstandard substratum, Speculat. Sci. Technol., 17(1994), 2-10.

7. HERRMANN, R.A. Operator equations, separation of variables and relativistic alterations, Intern. J. Math. Math. Sci., (to appear).

8. PROKNOVNIK, S.J. The Logic of Special Relativity, Cambridge University Press, London, 1967.

9. STROYAN, K.D. and W.A.J. LUXEMBURG, Introduction to the Theory of Infinitesimals, Academic Press, New York, 1976. 


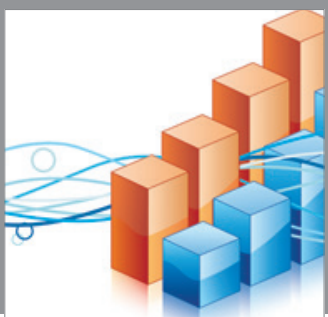

Advances in

Operations Research

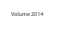

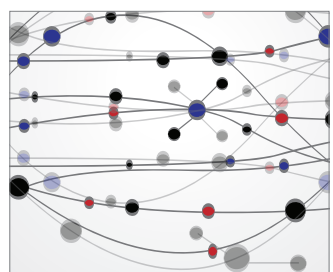

\section{The Scientific} World Journal
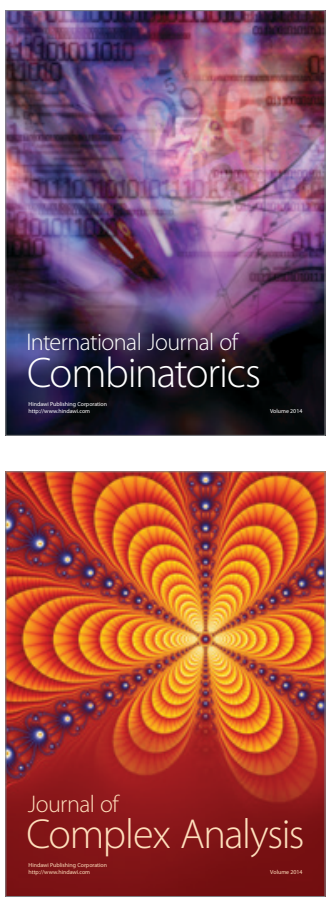

International Journal of

Mathematics and

Mathematical

Sciences
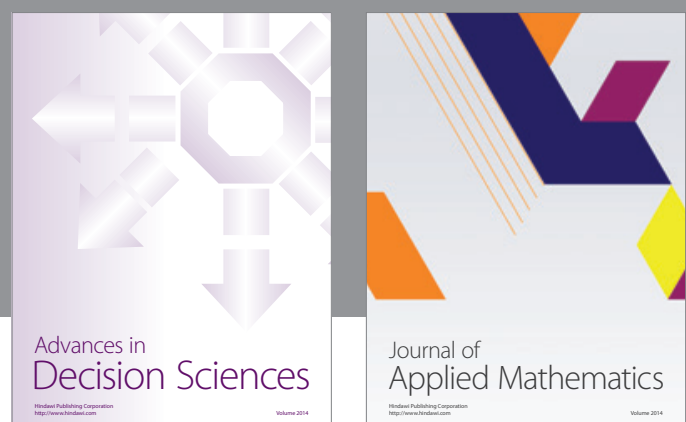

Journal of

Applied Mathematics
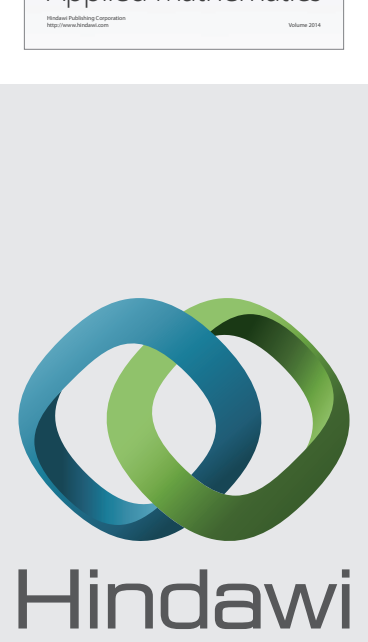

Submit your manuscripts at http://www.hindawi.com
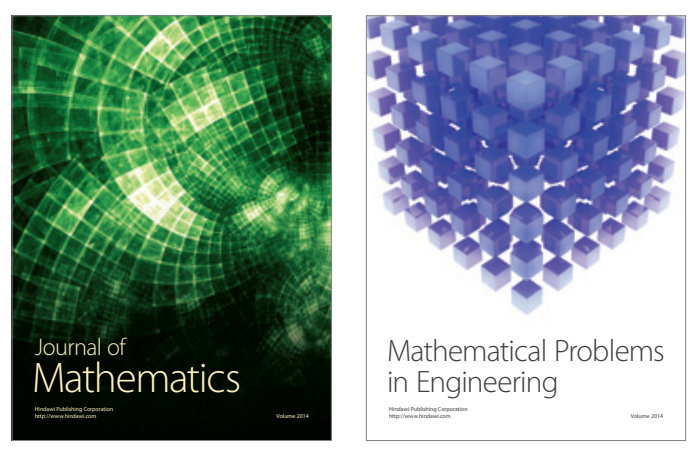

Mathematical Problems in Engineering
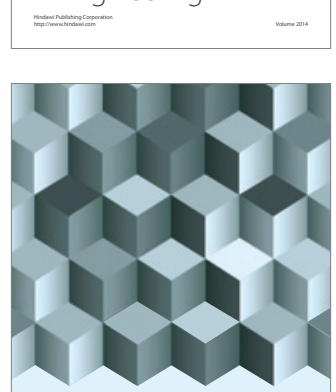

Journal of

Function Spaces
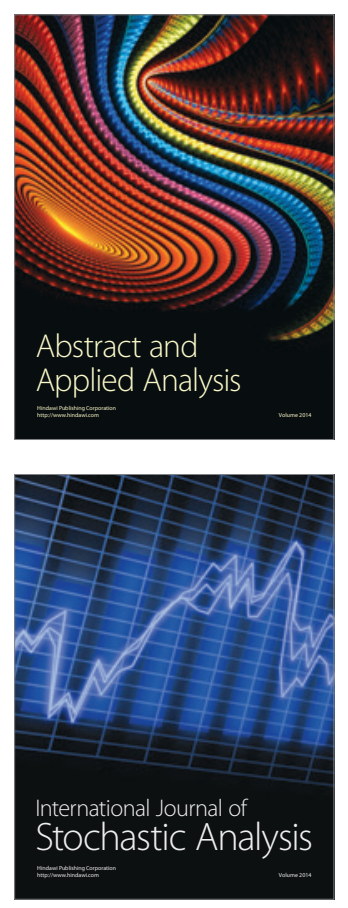

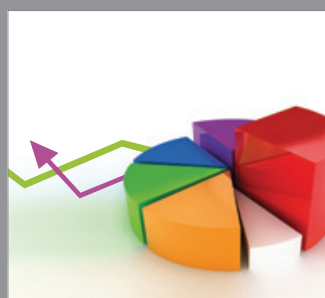

ournal of

Probability and Statistics

Promensencen
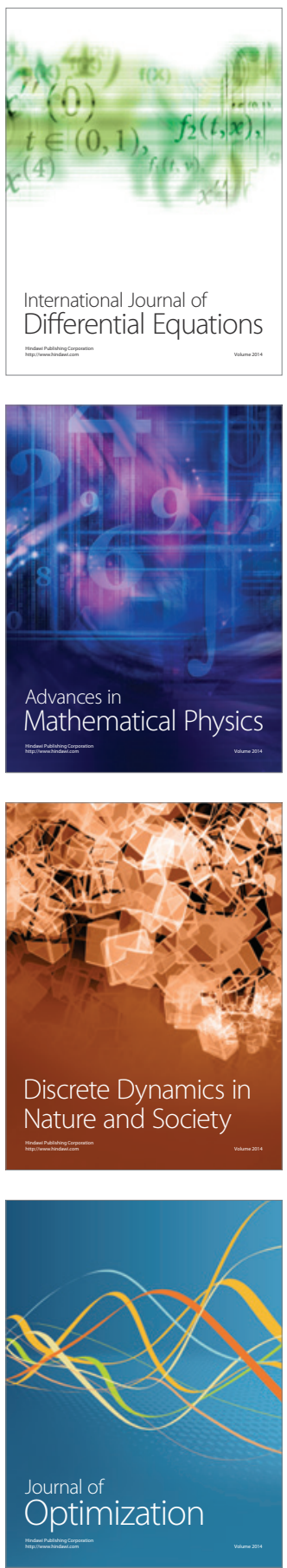